\title{
AOR
}

Selected Papers of \#AoIR2021:

The 22nd Annual Conference of the

Association of Internet Researchers Virtual Event / 13-16 Oct 2021

\section{REJECTING SCIENCE WITH SCIENCE: BOUNDARY-WORK IN ANTI-MASK TWITTER REPLY THREADS DURING COVID-19}

Andrew Beers

University of Washington, Human Centered Design \& Engineering

Sarah Nguyễn

University of Washington, Information School

Emma Spiro

University of Washington, Information School

Kate Starbird

University of Washington, Human Centered Design \& Engineering

\begin{abstract}
The COVID-19 pandemic has been marked by a controversy in the United States over the public health benefits of mask-wearing, especially on social media. Many have contested the scientific consensus that masks are an effective method to prevent and slow the spread of COVID-19 infections, often along explicitly political lines. Here, we investigate specifically how Twitter users engaging in arguments about mask-wearing invoke scientific principles to argue against masks. We further analyze the sources that these users cite to support their claims. Using a qualitative approach drawing from constructivist grounded theory, we show how these users work to defend the legitimacy of their claims and their external sources by selectively exploiting rhetorical values of scientific endeavour. We analogize their work to the process of scientific boundary-work, in which actors consciously manipulate the boundary between science and not-science for personal and political gain.
\end{abstract}

\section{Introduction}

In December 2020, Twitter announced updated rules meant to prevent medical misinformation during the COVID-19 pandemic [1]. These rules instituted several criteria

Suggested Citation (APA): Beers, A., Nguyễn, S., Spiro, E., Starbird, K. (2021 October). Rejecting Science with Science: Boundary-Work in Anti-Mask Twitter Reply Threads During COVID-19. Paper presented at AoIR 2021: The 22nd Annual Conference of the Association of Internet Researchers. Virtual Event: AoIR. Retrieved from http://spir.aoir.org. 
for the removal of misleading content on its platform, including being "demonstrably false or misleading" and "likely to impact public safety or cause serious harm." Exceptions to these rules include the "public debate about the advancement of COVID-19 science and research [...] so long as the claims don't misrepresent research findings." The strain between these two principles - misinformation and scientific debate - characterized the first year of the pandemic, where massive uncertainty of COVID-19's risks and remedies combined with widespread, durable, and politicized beliefs opposed mainstream scientific consensus [2]. Perhaps no element of the pandemic displays these tensions more than the public debate over mask-wearing. The mask debate was marked by disagreements and rapid changes of scientific consensus in its early stages, and a prolonged, political resistance to a pro-mask consensus that continues to this day.

In science, technology, and society studies, such rhetorical attempts to manage the margin between science and non-science have been termed by Gieryn as boundary-work [3]. Science's capaciousness - at times visionary and changeable, at other times conservative and immutable - allows rhetorical actors to selectively highlight certain attributes of science for political or personal gain. While Gieryn focused on rhetorical acts performed by expert scientists, we focus here on boundary-work done by Twitter users engaging in online debates about mask-wearing. We find that these users are well-attuned to the norms of scientific debate, and use several distinct rhetorical strategies to contest and claim the mantle of science in service of personal political causes.

\section{Methods}

Through Twitter's Streaming API, we captured all replies made to 73 accounts associated with United States governors and the mayor of the District of Columbia, due to these accounts' importance in setting statewide mask regulations. We selected two original (non-retweet) posts from each governor with the greatest number of mentions of the term 'mask' in the replies to that post, for 102 posts total. For each of these seed posts, we sampled 50 posts replying either directly to the governor, or to another user who had replied directly. Our final dataset contains 5,100 posts.

Drawing from Charmaz's method of constructivist grounded theory [4], we conducted an open coding process on this data. We focused on users' personal theories about mask effectiveness, and the citation behaviors behind these theories. During the coding process, we recorded qualitative memos on attributions and sourcing. These memos served as the basis for the following categorization of boundary work in mask discussions. 


\section{Findings}

We identify five rhetorical attributes of science that users in our dataset use to perform boundary-work: consensus, credentialing, randomized controlled trials, political neutrality, and consistency.

1. Anti-mask commenters understood the value of scientific consensus, even as their views contradicted the consensus reached by mainstream institutions. They shared alternative literature reviews to create the appearance of consensus, such as a frequently-cited quote tweet containing over 70 sources supposedly advocating against masks. Another oft-cited source is what Krafft and Donovan termed an evidence collage [5]: a screenshot of unknown origin, aggregating 12 mostly misleading quotes from 12 studies with the caption "ACCORDING TO MANY STUDIES MASKS DO NOT WORK" (Figure 1). Commenters shared these resources to frame pro-mask rhetoric as unscientific, despite these literature reviews often containing retracted, corrected, or contradictory scientific sources.

2. Anti-mask commenters were highly attuned to institutional credentials. Their most frequently-shared sources came from well-known and respected journals, such as a widely-shared (and later amended) editorial with anti-mask rhetoric published in the New England Journal of Medicine [6]. Credentialing proved most important in defending the often dubious histories of anti-mask scientists essential to the anti-mask movement. Commenters touted the medical expertise and credentials of these scientists and doctors, some of whom also weigh against scientific consensus on climate change and vaccines, to position them against what they perceived as non-scientific government officials mandating masks out of fear or ignorance.

3. Few rhetorical techniques were more powerful than the invocation of randomized controlled trials (RCT). Faced with the reality of an extensive pro-mask scientific literature, anti-mask users undermined these studies by claiming the experiments were not RCT, but rather only observational studies or literature reviews. One user shared an article criticizing pro-mask scientific studies with the comment that "tests not done with RCT is like TSA security theatre, we only THINK it helps." Anti-mask commenters followed the lead of the anti-mask doctors and scientists, who produced videos critiquing pro-mask sources often specifically for not being RCT. 
4. Despite anti-mask commenters being avid political partisans, they prized the principle of political neutrality. One user sums up this predominant rhetorical strategy of the anti-mask movement: "Technically, pre covid, all studies said masks don't work. The science didn't change, politics did." Anti-mask users decried scientists and politicians alike for citing science compromised by political bias or ulterior motives, and used these claims to discredit many pro-mask claims. A particular target was National Institute of Allergy and Infectious Diseases director Dr. Anthony Fauci, one of the U.S.'s foremost scientists and advocates of mask-wearing. Accused of harboring anti-Trump bias, or aims towards a secret eugenics program, Dr. Fauci's claims were routinely dismissed.

5. When faced with compelling evidence in favor of masks, anti-mask users exploited the principle of consistency. The first month of the pandemic was marred by conflicting statements from scientists and politicians as to the efficacy of masks, although scientific consensus became pro-mask soon after. Anti-mask commenters used this period of uncertainty to dismiss and distrust the reports of any source which had formerly been anti-mask. One of the most shared YouTube videos by anti-mask users was a video of Dr. Fauci denigrating mask-wearing, used as evidence that his later pro-mask claims should be disregarded. We note that pro-mask commenters did their own boundary-work by explaining the merits of scientific progress, and how knowledge accumulates progressively over time.

\section{Acknowledgements}

This research was supported by the National Science Foundation COVID-19 Rapid Response Research (NSF RAPID) program (2027792). We also wish to thank UW Center for an Informed Public for providing infrastructure support.

\section{References}

[1] "COVID-19 misleading information policy," 2020.

https://help.twitter.com/en/rules-and-policies/medical-misinformation-policy

(accessed Jan. 11, 2021).

[2] J. S. Brennen, F. M. Simon, P. N. Howard, and R. K. Nielsen, "Types, Sources, and Claims of COVID-19 Misinformation," p. 13, 2020.

[3] T. F. Gieryn, "Boundary-Work and the Demarcation of Science from Non-Science: Strains and Interests in Professional Ideologies of Scientists," Am. Sociol. Rev., vol. 
48, no. 6 , pp. $781-795,1983$, doi: $10.2307 / 2095325$.

[4] A. Bryant and K. Charmaz, "Asking Questions of the Data: Memo Writing in the Grounded Theory Tradition," in The SAGE Handbook of Grounded Theory, SAGE, 2007.

[5] P. M. Krafft and J. Donovan, "Disinformation by Design: The Use of Evidence Collages and Platform Filtering in a Media Manipulation Campaign," Polit. Commun., vol. 37, no. 2, pp. 194-214, Mar. 2020, doi: 10.1080/10584609.2019.1686094.

[6] M. Klompas, C. A. Morris, J. Sinclair, M. Pearson, and E. S. Shenoy, "Universal Masking in Hospitals in the Covid-19 Era," N. Engl. J. Med., vol. 382, no. 21, p. e63, May 2020, doi: 10.1056/NEJMp2006372.

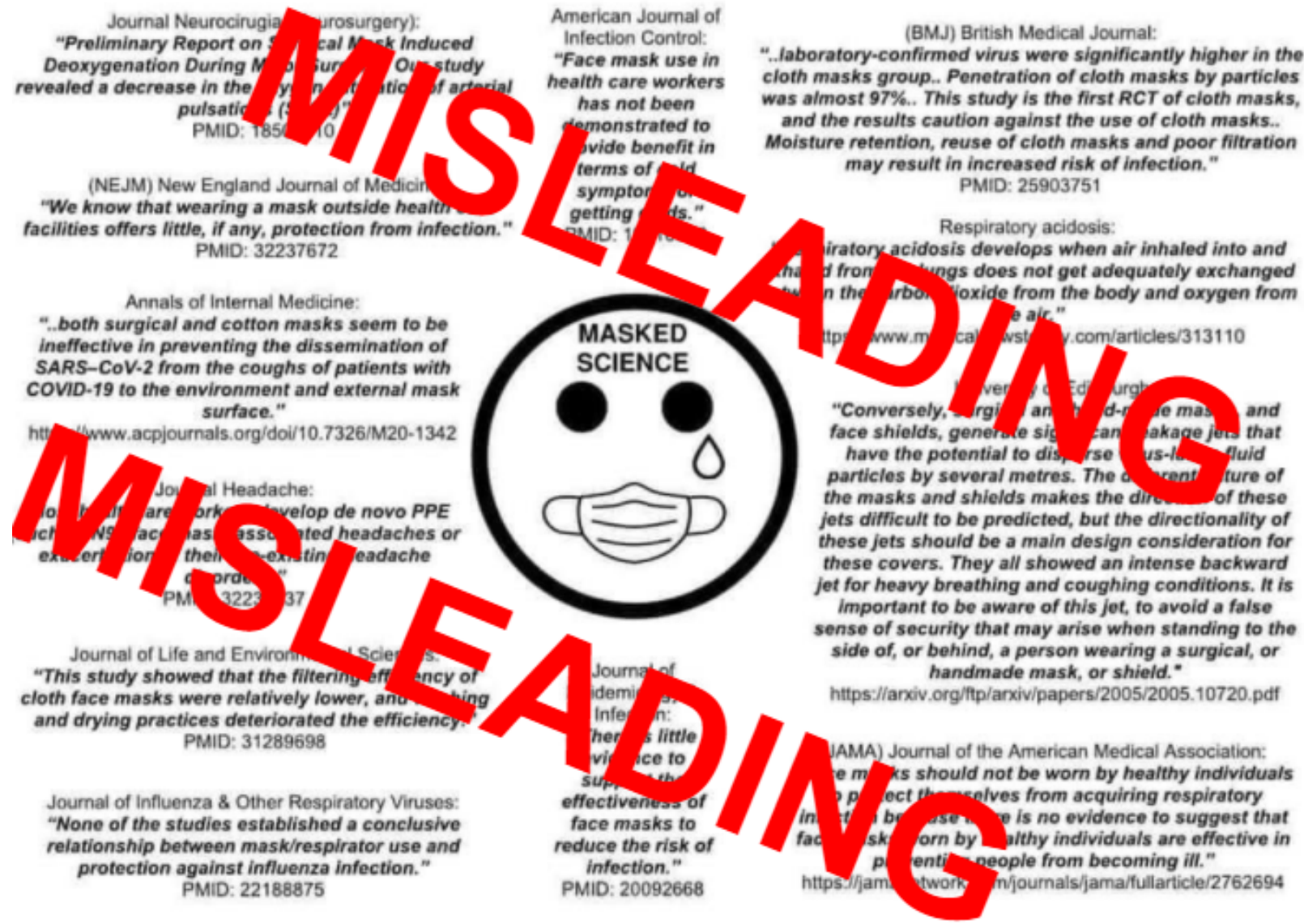


Figure 1: An example of an evidence collage that suggests a false scientific consensus that masks are not effective in mitigating the spread of coronavirus. The source is unknown. "MISLEADING" text added by authors. 